\title{
The multiplication groups of topological loops
}

\author{
Ágota Figula
}

\begin{abstract}
In this short survey we discuss the question which Lie groups can occur as the multiplication groups $M u l t(L)$ of connected topological loops $L$ and we describe the correspondences between the structure of the group $\operatorname{Mult}(L)$ and the structure of the loop $L$.
\end{abstract}

2010 Mathematics Subject Classification: 57S20, 22E25, 20N05, 57M60

Key words and phrases: multiplication group of loops, topological transformation group, solvable Lie groups, filiform Lie groups

\section{Introduction}

A loop $(L, \cdot)$ is a quasigroup with identity element $e \in L$. The left translations $\lambda_{a}: y \mapsto a \cdot y$ and the right translations $\rho_{a}: y \mapsto y \cdot a, a \in L$, are bijections of $L$. To obtain closer relations between a loop and a group one has to investigate the groups which are generated by the translations of a loop $L$. The group generated by all left and right translations of $L$ is called the multiplication group $\operatorname{Mult}(L)$ of $L$ (cf. [1], [2]). The subgroup $G$ of $\operatorname{Mult}(L)$ generated by all left translations of $L$ is the group of left translations of $L$. The group $M$ ult $(L)$ reflects well the normal structure of the corresponding loop $L$, since for any normal subloop of $L$ there is a normal subgroup in $M u l t(L)$ and for every normal subgroup $N$ of $M u l t(L)$ the orbit $N(e)$ is a normal subloop of $L$. Hence, it is an interesting question which groups can be represented as multiplication groups of loops. The criterion for the decision whether a group is the multiplication group of a loop $L$ is given in [16]. This criterion has been successfully applied in particular in the case of Lie groups.

In [15] topological and differentiable loops $L$ having a Lie group as the group $G$ of left translations of $L$ are studied. There the topological loops $L$ are treated as continuous sharply transitive sections $\sigma: G / H \rightarrow G$, where $H$ is the stabilizer of $e \in L$ in $G$. Publications, in which classes of connected topological loops $L$ have been classified (cf. [15], [5]) show that there are only few Lie groups which are not groups of left translations of a topological loop. If $L$ is a topological loop having a Lie group as the group of left translations, then the group $\operatorname{Mult}(L)$ is prevalently a differentiable transformation group of infinite dimension. The condition for $\operatorname{Mult}(L)$ to be a Lie group is a 
strong restriction. Namely, every 1-dimensional topological loop having a Lie group as its multiplication group is already a group ([15], Theorem 18.18, p. 248). For 2-dimensional topological loops $L$ the group $M u l t(L)$ is a Lie group precisely if it is an elementary filiform Lie group of dimension $\geq 4$ ([4], Theorem 1, p. 420). In contrast to this many 3-dimensional connected topological loops have nilpotent but not filiform as well as solvable but not nilpotent multiplication group. The aim of this talk is to present the most important recent results in this direction.

\section{Preliminaries}

A binary system $(L, \cdot)$ is called a loop if there exists an element $e \in L$ such that $x=e \cdot x=x \cdot e$ holds for all $x \in L$ and the equations $a \cdot y=b$ and $x \cdot a=b$ have precisely one solution, which we denote by $y=a \backslash b$ and $x=b / a$. A loop $L$ is proper if it is not a group.

The left and right translations $\lambda_{a}: y \mapsto a \cdot y: L \times L \rightarrow L$ and $\rho_{a}$ : $y \mapsto y \cdot a: L \times L \rightarrow L, a \in L$, are bijections of $L$. The permutation group $\operatorname{Mult}(L)$ generated by all left and right translations of the loop $L$ is called the multiplication group of $L$ and the stabilizer of $e \in L$ in the group $\operatorname{Mult}(L)$ is called the inner mapping group $\operatorname{Inn}(L)$ of $L$.

An important problem is to be analyzed under which circumstances a group is the multiplication group of a loop. The answer to this question was given in [16], Theorem 4.1, which says that the multiplication group of a loop can be characterized in purely group theoretic terms by using the notion of connected transversals. Let $K$ be a group, let $S \leq K$, and let $A$ and $B$ be two left transversals to $S$ in $K$. We say that $A$ and $B$ are $S$-connected if $a^{-1} b^{-1} a b \in S$ for every $a \in A$ and $b \in B$. The core $C o_{K}(S)$ of $S$ in $K$ is the largest normal subgroup of $K$ contained in $S$.

Theorem 1. A group $K$ is isomorphic to the multiplication group of a loop if and only if there exists a subgroup $S$ with $C_{K}(S)=1$ and $S$-connected transversals $A$ and $B$ satisfying $K=\langle A, B\rangle$.

In this theorem the subgroup $S$ is the inner mapping group of $L$ and $A$ and $B$ are the sets of left and right translations of $L$, respectively.

The kernel of a homomorphism $\alpha:(L, \cdot) \rightarrow\left(L^{\prime}, *\right)$ of a loop $L$ into a loop $L^{\prime}$ is a normal subloop $N$ of $L$. The centre $Z(L)$ of a loop $L$ consists of all elements $z$ which satisfy the equations $z x \cdot y=z \cdot x y, x \cdot y z=x y \cdot z, x z \cdot y=$ $x \cdot z y, z x=x z$ for all $x, y \in L$. If we put $Z_{0}=e, Z_{1}=Z(L)$ and $Z_{i} / Z_{i-1}=Z\left(L / Z_{i-1}\right)$, then we obtain a series of normal subloops of $L$. If $Z_{n-1}$ is a proper subloop of $L$ but $Z_{n}=L$, then $L$ is centrally nilpotent of class $n$. In [2] it was proved that if $\operatorname{Mult}(L)$ is a nilpotent group of class $n$, then $L$ is centrally nilpotent of class at most $n$. The next assertion gives the connections between the normal subgroup structure of $\operatorname{Mult}(L)$ and the normal subloop structure of $L$. 
Lemma 2. Let $L$ be a loop with multiplication group $M u l t(L)$ and identity element $e$.

(i) Let $\alpha$ be a homomorphism of the loop $L$ onto the loop $\alpha(L)$ with kernel $N$. Then $N$ is a normal subloop of $L$ and $\alpha$ induces a homomorphism of the group Mult $(L)$ onto the group $\operatorname{Mult}(\alpha(L))$.

Let $M(N)$ be the set $\{m \in M u l t(L) ; x N=m(x) N$ for all $x \in L\}$. Then $M(N)$ is a normal subgroup of Mult $(L)$ containing the multiplication group $M u l t(N)$ of the loop $N$ and the multiplication group of the factor loop $L / N$ is isomorphic to Mult $(L) / M(N)$.

(ii) For every normal subgroup $\mathcal{N}$ of $M$ ult $(L)$ the orbit $\mathcal{N}(e)$ is a normal subloop of $L$. Moreover, $\mathcal{N} \leq M(\mathcal{N}(e))$.

The proof of Lemma 2 can be found in [1], Theorems 3, 4 and 5 and in [3], IV.1, Lemma 1.3.

A loop $L$ is called topological if $L$ is a topological space and the binary operations $(x, y) \mapsto x \cdot y,(x, y) \mapsto x \backslash y,(x, y) \mapsto y / x: L \times L \rightarrow L$ are continuous. Let $G$ be a connected Lie group, $H$ be a subgroup of $G$. A continuous section $\sigma: G / H \rightarrow G$ is called continuous sharply transitive section, if the set $\sigma(G / H)$ operates sharply transitively on $G / H$, which means that to any $x H$ and $y H$ there exists precisely one $z \in \sigma(G / H)$ with $z x H=y H$. Every connected topological loop $L$ having a Lie group $G$ as the group of left translations of $L$ is obtained on a homogeneous space $G / H$, where $H$ is a closed subgroup of $G$ with $C o_{G}(H)=1$ and $\sigma: G / H \rightarrow G$ is a continuous sharply transitive section such that $\sigma(H)=1 \in G$ and the subset $\sigma(G / H)$ generates $G$. The multiplication of $L$ on the manifold $G / H$ is defined by $x H * y H=\sigma(x H) y H$. Moreover, the subgroup $H$ is the stabilizer of the identity element $e \in L$ in the group $G$. The following assertion is proved in [9], IX.1.

Remark 3. For any connected topological loop there exists universal covering which is simply connected.

A quasi-simple connected Lie group is a connected Lie group $G$ such that any normal subgroup of $G$ is discrete and central in $G$. A semi-simple connected Lie group $G$ has the form $G=G_{1} \cdot G_{2} \cdots G_{r}$, where $G_{i}$ are normal quasi-simple connected Lie subgroups such that $G_{i} \cap G_{j}$ is a discrete central subgroup of $G$. A connected loop $L$ is quasi-simple if any normal subloop of $L$ is discrete in $L$. According to [9], p. 216, all discrete normal subloops of a connected loop are central.

The elementary filiform Lie group $\mathcal{F}_{n}$ is the simply connected Lie group of dimension $n \geq 3$ such that its Lie algebra has a basis $\left\{e_{1}, \cdots, e_{n}\right\}$ with $\left[e_{1}, e_{i}\right]=e_{i+1}$ for $2 \leq i \leq n-1$. A 2-dimensional simply connected loop $L_{\mathcal{F}}$ is called an elementary filiform loop if its multiplication group is an elementary filiform group $\mathcal{F}_{n}, n \geq 4([6])$.

Homogeneous spaces of solvable Lie groups are called solvmanifolds. 


\section{Multiplication groups of topological loops of dimension $\leq 3$}

If $L$ is a connected topological loop having a Lie group as its multiplication group $\operatorname{Mult}(L)$, then $\operatorname{Mult}(L)$ acts transitively and effectively as a topological transformation group on $L$. All transitive transformation groups on a manifold of dimension $\leq 2$ have been classified by Lie (cf. [10]) and Mostow in [11], $\S 10$, pp. 625-635.

Every topological loop $L$ which is realized on a connected 1-manifold is homeomorphic either to the real line or to the circle $S^{1}$. If the group $G$ topologically generated by the left translations of $L$ is locally compact, then $G$ is a finite covering of the group $P S L_{2}(\mathbb{R})$ if $L$ is a circle and $G$ is the universal covering of $P S L_{2}(\mathbb{R})$ if $L$ is a line. Every covering of $P S L_{2}(\mathbb{R})$ occurs for a suitable $L$ (cf. [15], Propositions 18.1, 18.2, p. 235). But in [15], Theorem 18.18, p. 248, it is proved that there does not exist 1-dimensional proper topological loop $L$ such that the multiplication group of $L$ is locally compact.

If $L$ has dimension 2, then among the 3 -dimensional solvable Lie groups there are only two groups which are not groups of left translations of $L$. These are the connected component of the group of motions or the connected component of the group of homotheties of the Euclidean plane (cf. [5]). In contrast to this there is only one type of Lie groups which are multiplication groups of $L$. These are the elementary filiform Lie groups as Theorem 1 in [4], p. 420, shows:

Theorem 4. Let $L$ be a proper connected simply connected 2-dimensional topological loop. The multiplication group $\operatorname{Mult}(L)$ of $L$ is a Lie group if and only if $\operatorname{Mult}(L)$ is an elementary filiform Lie group $\mathcal{F}_{n}$ with $n \geq 4$. Moreover, the group $G$ of left translations of $L$ is an elementary filiform Lie group $\mathcal{F}_{m}$, where $3 \leq m \leq n$, and the inner mapping group $\operatorname{Inn}(L)$ of $L$ is abelian.

The proof of Theorem 4 is based on the classification of Mostow (cf. [11], $\S 10)$. The loop $L$ of Theorem 4 is a central extension of the group $\mathbb{R}$ by the group $\mathbb{R}$ (cf. Theorem 28.1 in [15], p. 338). Hence it is a centrally nilpotent loop of class 2 and can be represented in $\mathbb{R}^{2}$. If $L$ is not simply connected but satisfies all other conditions of Theorem 4, then $L$ is homeomorphic to the cylinder $\mathbb{R} \times \mathbb{R} / \mathbb{Z}$. In this case the multiplication group $\operatorname{Mult}(L)$ of $L$ is a Lie group of dimension $n \geq 4$ with elementary filiform Lie algebra such that the centre of $\operatorname{Mult}(L)$ is isomorphic to the group $\mathrm{SO}_{2}(\mathbb{R})$ (cf. Theorem 4 and Theorem 28.1 in [15], p. 338).

Now let $L$ be a 3-dimensional connected simply connected topological loop having a Lie group as its multiplication group. As there does not exist a multiplication with identity on the sphere $S^{2}$ the loop $L$ is homeomorphic to $\mathbb{R}^{3}$ or to $S^{3}$ (cf. [8], p. 210). 
A transitive action of a connected Lie group $G$ on a manifold $M$ is called minimal, if it is locally effective and if $G$ does not contain subgroups acting transitively on $M$. The minimal actions of non-solvable Lie groups on 3dimensional manifolds are given in [8], Table 1, p. 201.

Theorem 5. There does not exist proper connected topological loop $L$ of dimension 3 such that its multiplication group $\operatorname{Mult}(L)$ is a non-solvable Lie group acting minimally on the manifold $L$.

The proof of Theorem 5 can be found in [6], Theorem 3.1, p. 388. If $L$ is a 3-dimensional quasi-simple connected simply connected topological loop, then the following non-solvable Lie groups can occur as the multiplication group of $L$ (cf. Proposition 3.2 in [6], p. 389):

Proposition 6. Let $L$ be a 3-dimensional quasi-simple connected simply connected topological loop such that the multiplication group Mult $(L)$ of $L$ is a Lie group.

(a) If $L$ is homeomorphic to $S^{3}$, then the group $M u l t(L)$ is either isomorphic to the semidirect product $\operatorname{Spin}_{3}(\mathbb{R}) \rtimes S O_{3}(\mathbb{R})$ or it is one of the following quasi-simple Lie groups: $S O_{5}(\mathbb{R}, 1), S U_{3}(\mathbb{C}, 1), S L_{4}(\mathbb{R}), S L_{2}(\mathbb{C}), S p_{4}(\mathbb{R})$, the universal covering of $S L_{3}(\mathbb{R})$.

(b) If $L$ is homeomorphic to $\mathbb{R}^{3}$, then one of the following holds:

(i) If $M u l t(L)$ is quasi-simple, then it is the group $P S L_{2}(\mathbb{C})$.

(ii) If Mult $(L)$ is semi-simple, then it is isomorphic to the semidirect product $P S L_{2}(\mathbb{R}) \rtimes P S L_{2}(\mathbb{R})$.

(iii) If $M$ ult $(L)$ is not semi-simple, then it is the semidirect product $\mathbb{R}^{3} \rtimes$ $S$, where $S$ is isomorphic either to $S O_{3}(\mathbb{R})$ or to $S L_{3}(\mathbb{R})$ respectively to $P S L_{2}(\mathbb{R})$ and acts irreducibly on $\mathbb{R}^{3}$.

As $S^{3}$ is not a solvmanifold (cf. [12]) every 3-dimensional proper connected simply connected topological loop which has a solvable Lie group as its multiplication group is homeomorphic to $\mathbb{R}^{3}$. Till now no 3 -dimensional locally compact connected loop $L$ has been found having a filiform group $\mathcal{F}_{n}$ as its multiplication group $M u l t(L)$. In contrast to this there is a plethora of loops $L$ for which $\operatorname{Mult}(L)$ is a nilpotent but not filiform Lie group.

Theorem 7. Among the nilpotent Lie groups the direct products $\mathcal{F}_{n} \times{ }_{Z} \mathcal{F}_{m}$ of the elementary filiform Lie groups $\mathcal{F}_{n}, \mathcal{F}_{m}$ with amalgamated center $Z$ such that $(n, m) \in \mathbb{N} \backslash\{(3,3)\}$ as well as the groups $\mathbb{R} \times \mathcal{F}_{n}$ with $n \geq 4$ are multiplication groups of 3-dimensional simply connected topological loops.

The proof of this assertion can be found in [6], Propositions 4.3 and 5.1. Every loop $L$ of Theorem 7 having a Lie group $\mathcal{F}_{n} \times{ }_{Z} \mathcal{F}_{m}$ as the multiplication group $\operatorname{Mult}(L)$ is a central extension of the group $\mathbb{R}$ by the group $\mathbb{R}^{2}$. Each 
loop $L$ of Theorem 7 having a direct product $\mathcal{F}_{n} \times \mathbb{R}$ as the multiplication group $\operatorname{Mult}(L)$ is a central extension of the group $\mathbb{R}^{2}$ by the group $\mathbb{R}$.

In contrast to the 2-dimensional topological loops there are many 3dimensional loops with solvable but non-nilpotent multiplication group. In [7] we have classified the solvable non-nilpotent Lie groups of dimension $\leq 5$ which are multiplication groups for 3-dimensional simply connected topological loops $L$. Using the classification of solvable Lie algebras given in [13], [14] and the relations between the normal subloops of $L$ and the normal subgroups of $M u l t(L)$ (cf. Lemma 2) we have proved that each 3dimensional simply connected topological loop $L$ having a solvable Lie group of dimension $\leq 5$ as the multiplication group of $L$ is a central extension of the group $\mathbb{R}$ by $\mathbb{R}^{2}$. Therefore the group $\operatorname{Mult}(L)$ is a semidirect product of the abelian group $M$ by a group $Q \cong \mathbb{R}^{2}$ such that $M=Z \times \operatorname{Inn}(L)$, where $\mathbb{R} \cong Z$ is a central subgroup of $\operatorname{Mult}(L)$ and $\operatorname{dim} \operatorname{Inn}(L) \in\{1,2\}$. Applying this we prove the following theorem.

Theorem 8. Let $L$ be a connected simply connected topological proper loop of dimension 3 such that its multiplication group is an at most 5-dimensional solvable non-nilpotent Lie group. Then the following Lie groups are the multiplication groups $M u l t(L)$ of $L$ :

If Mult $(L)$ has 1-dimensional centre, then it is either the group $\mathcal{F}_{3} \times \mathcal{L}_{2}$, where $\mathcal{L}_{2}$ is the non-abelian 2-dimensional simply connected Lie group, or the direct product $\mathbb{R} \times \Sigma$, where $\Sigma$ is one of the following Lie groups: $\Sigma=\mathcal{L}_{2} \times \mathcal{L}_{2}$ or $\Sigma$ is the 4-dimensional indecomposable solvable Lie group which has no 1-dimensional normal subgroup or $\Sigma$ is the 4-dimensional solvable Lie group which has trivial centre, 2-dimensional commutator subgroup and precisely one 1-dimensional normal subgroup.

If $\operatorname{Mult}(L)$ has 2-dimensional centre, then it is the direct product of the group $\mathbb{R}^{2}$ and a 3-dimensional Lie group having 2-dimensional commutator subgroup.

\section{Acknowledgement}

The research was supported by the Hungarian Scientific Research Fund (OTKA) Grant PD 77392 and by the János Bolyai Research Fellowship.

\section{References}

[1] A. A. Albert, Quasigroups I. Trans. Amer. Math. Soc. 54 (1943), 507519 .

[2] R. H. Bruck, Contributions to the theory of loops. Trans. Amer. Math. Soc. 60 (1946), 245-354.

[3] R. H. Bruck, A Survey of binary systems. Springer-Verlag, Berlin, Heidelberg, New York, 1971. 
[4] Á. Figula, The multiplication groups of 2-dimensional topological loops. J. Group Theory 12 (2009), 419-429.

[5] Á. Figula, Topological loops with three-dimensional solvable left translation group. Aequat. Math. 79 (2010), 83-97.

[6] Á. Figula, On the multiplication groups of three-dimensional topological loops. J. Lie Theory 21 (2011), 385-415.

[7] Á. Figula, Three-dimensional topological loops with solvable multiplication groups. submitted for publication (2011), pp. 34 .

[8] V. V. Gorbatsevich, On three-dimensional homogeneous spaces. Sib. Math. Zh. 18 (1977), no. 2, 280-293. English transl.: Sib. Math. J. 18, 200-210.

[9] K. H. Hofmann, K. Strambach, Topological and analytical loops. Quasigroups and Loops: theory and applications, 205-262, Sigma Ser. Pure Math., 8, Heldermann, Berlin, 1990.

[10] S. Lie, F. Engels, Theorie der Transformationsgruppen. Band 3. Verlag von B.G. Teubner, Leipzig, 1893.

[11] G. D. Mostow, The extensibility of local Lie groups of transformations and groups of surfaces. Ann. of Math. (2) $\mathbf{5 2}$ (1950), 606-636.

[12] G. D. Mostow, Factor spaces of solvable groups. Ann. of Math. (2) 60 (1954), 1-27.

[13] G. M. Mubarakzjanov, On solvable Lie algebras. (Russian) Izv. Vyssh. Uchebn. Zaved. Matematika 1963, No. 1 (32), 114-123.

[14] G. M. Mubarakzjanov, Classification of real structures of Lie algebras of fifth order. (Russian) Izv. Vyssh. Uchebn. Zaved. Matematika 1963, No. 3 (34), 99-106.

[15] P. T. Nagy, K. Strambach, Loops in group theory and Lie theory. de Gruyter Expositions in Mathematics, 35. Walter de Gruyter, Berlin, 2002.

[16] M. Niemenmaa, T. Kepka, On multiplication groups of loops. J. Algebra 135 (1990), No. 1, 112-122.

Author's address:

Institute of Mathematics University of Debrecen

H-4010 Debrecen, P.O.B. 12

Hungary

E-mail: figula@science.unideb.hu 